Estudiante de Licenciatura en Ciencia Política

(UNR). E-mail: vanidemoises@hotmail.com

\title{
Daniela Ingaramo
}

Estudiante de Licenciatura en Ciencia Política (UNR). E-mail: daningaramo@hotmail.com.ar

\section{Gabriel Dileo}

Lic. en Trabajo Social (UNR). Dirección Provincial de Promoción de Derechos Niñez, Adolescencia y Familia, Ministerio de Desarrollo Social, Provincia de Santa Fe. E-mail:

analia_gabriel@yahoo.com.ar

\section{Romina Lusardi}

Lic. en Trabajo Social (UNR).

Centro de Desarrollo Zonal $N^{\circ} 7$, Ministerio de Desarrollo Social, Provincia de Santa Fe. E-mail: rominalusardi@hotmail.com

\section{La implementación del PFIC en Rosario, obstáculos y logros ${ }^{1}$}

Resumen

El presente trabajo relata la experiencia del equipo encargado de la implementación del Programa de Fortalecimiento de Iniciativas Comunitarias en la ciudad de Rosario, su vínculo con las instituciones, la población destinataria y las dificultades encontradas en este proceso. El objetivo es contribuir al mejoramiento del desarrollo y el impacto de programas similares, teniendo como fin generar información útil para los decisores y ejecutores.

\section{Palabras clave}

fortalecimiento · implementación · equipo · obstáculos

\section{Introducción}

La realización del presente trabajo surge a raíz de la necesidad de volcar las inquietudes que fueron surgiendo en la puesta en práctica del Programa de Fortalecimiento de Iniciativas Comunitarias (en adelante PFIC) en la ciudad de Rosario. Teniendo en cuenta la utilidad que puede presentar la elaboración de este escrito, consideramos que, por un lado, nos lo debemos como grupo en tanto, quizá, sea el único registro que quede de nuestro trabajo, dadas nuestras condiciones laborales y nuestra inexistencia en la estructura provincial; una labor que si bien encontró obstáculos importantes en su desarrollo, fue hecha con la mayor calidad, responsabilidad y empeño dentro de las posibilidades y medios con que contamos. Por el otro, permitirá explicitar ciertos ajustes necesarios para facilitar que futuras actividades similares al Programa analizado, se hagan de manera oportuna, en forma eficiente y con la calidad esperada.

Otras dos cuestiones que no debemos dejar de abordar son el modo de desempeñarse dentro del ámbito público y el vínculo que se establece con los destinatarios del Programa, porque nuestra experiencia nos demostró que es posible otra forma de trabajo y otro trato hacia la gente dentro de este ámbito, donde se tenga en cuenta el esfuerzo que estas personas realizan, y sean respetadas como tales.

${ }^{1}$ PFIC: Programa de Fortalecimiento de Iniciativas Comunitarias. 
Creemos que un proceso evaluativo que tenga en cuenta las percepciones de todos los involucrados puede promover cambios en los reglamentos y en las prácticas cotidianas, que enriquezcan la implementación de este tipo de acciones. De esta forma, los resultados finales serán mejores, tanto en cantidad como en calidad.

\section{¿Qué entendemos por evaluación?}

Compartimos una concepción de la política como herramienta de transformación de la sociedad, como un instrumento que posibilita el cambio, en el sentido de lograr mejores condiciones de vida para la población. También consideramos que la praxis política debe tener una fuerte carga ética, que debe aplicarse y desplegarse en la labor cotidiana.

Basándonos en esta concepción intentamos llevar a cabo la ejecución del PFIC en la ciudad de Rosario. El objeto de esta evaluación es lograr que muchas de las dificultades encontradas puedan superarse en la implementación futura de programas similares.

En este marco, la evaluación debe proporcionar información que sea creíble y útil, para permitir la incorporación de la experiencia adquirida en el proceso de adopción de decisiones. Forma parte integral del proceso de gestión, no es un fin en sí mismo, tiene el fin de generar información útil para los decisores y ejecutores.

El proceso de evaluación procura determinar la relevancia, eficacia, eficiencia e impacto de un proyecto o programa, a la luz de sus objetivos. Es una actividad gerencial, en este caso interna, que analiza las relaciones de los procesos y recursos con los resultados e impactos. Esta evaluación es un análisis retrospectivo de cuestiones operativas, procesos y cuestiones administrativas, en el cual se identifican problemas dentro del programa que lo alejan de los objetivos perseguidos.

El informe presenta el seguimiento de las acciones que formaron parte del Programa así como los resultados de las mismas, buscando determinar el grado en que el desenlace coincide con lo programado, con el fin de detectar deficiencias, obstáculos y/o necesidades de ajuste en la ejecución.

\section{Descripción del programa}

a) Origen

El PFIC surge en el año 2006, a través del decreto $N^{\circ}$ 1850/06 del gobierno de la Provincia de Santa Fe, durante la gestión del Ing. Jorge Obeid, quedando designado como órgano ejecutor la Secretaría de Estado de Promoción Comunitaria y, dentro de ella, la Unidad Evaluadora de Proyectos Sociales.

En el año 1995 se sancionó la ley nacional n²4.443, de creación del Fondo de Emergencia Social para el conurbano de las ciudades de Santa Fe y Rosario. A partir de su sanción, dicha ley permitió a la Provincia de Santa Fe recibir la suma de hasta 30 millones de pesos por año de los recursos que le correspondían a la provincia de Buenos Aires en virtud del art. 19 inc. b) de la ley 23.966.

Tomando como antecedente el fondo del conurbano bonaerense se llegó a 
la sanción de esta ley, que encontraba sus fundamentos en el proceso de migración interna por el cual grandes contingentes humanos provenientes de diferentes provincias argentinas se asentaron en el cordón de las ciudades más importantes del Litoral y/o Gran Buenos Aires.

En su artículo primero, la ley establece el destino que los fondos pueden tener, habiéndose orientado con el transcurso de los años hacia obras de infraestructura y asistencia social, canalizado a través de municipios, comunas y ONGs, de los conurbanos de las ciudades de Santa Fe y Rosario. El Programa de Fortalecimiento fue creado a partir de estos fondos.

b) Características

El Programa consistió en la entrega de subsidios no reintegrables, destinados a capacitación, infraestructura, equipamiento, insumos y asistencia técnica, a ONGs sin fines de lucro, Municipios y Comunas; estuvo destinado a las localidades de Santa Fe, Rosario, San Lorenzo, Casilda, Desvío Arijón, Coronda y Villa Constitución y comprendió cuatro rubros a financiar: 1)Equipa miento (mobiliario para el desarrollo del proyecto); 2) Bienes (insumos para el desarrollo del proyecto); 3) Servicios (obras menores de infraestructura, cuyo financiamiento corresponde a ampliación y/o refacción consideradas esenciales para el desarrollo del proyecto); 4) Servicios técnico-profesionales (acciones de capacitación técnica específica cuyos recursos humanos sean requeridos como apoyo a la organización en el desarrollo del proyecto; contiene tres categorías: profesionales -quienes cuentan con título universitario-, técnicos quienes acreditan título terciario- y promotores -aquellos que realizaron cursos o talleres informales de la actividad en que se desarrollarán como capacitadores).

c) Objetivos

El objetivo del Programa fue «contribuir con las ONGs, Municipios y Comunas para que éstas desarrollen acciones en la comunidad, promoviendo la participación comunitaria y el desarrollo local, a través de: desarrollo deportivo, educativo y cultural, prevención y promoción de la salud, servicios a la comunidad, saneamiento ambiental, atención de niños, adolescentes, jóvenes, familias y tercera edad». En su sentido más amplio, el Programa de referencia apuntó a fortalecer las Iniciativas Comunitarias que tendieran a la prevención, promoción y contención de grupos vulnerables y revalorizar el accionar de las mismas en el ámbito comunitario.

d) Destinatarios

El Programa estuvo destinado a las organizaciones de la sociedad civil sin fines de lucro, con personería jurídica otorgada por la Dirección General de Personas Jurídicas (Asociaciones Civiles, Fundaciones, Cooperadoras, Cooperativas, Mutuales, etc.) cuya actividad de base estuviera orientada a la promoción y desarrollo comunitario. Aquellas organizaciones que no contaban con personería jurídica podían recibir el subsidio a través de su asociatividad con otras organizaciones que sí la poseyeran, siendo estas últimas las responsables. También estuvo dirigido a Municipios y Comunas de la Provincia de Santa Fe.

e) Financiamiento

Los fondos utilizados para llevar a cabo el Programa tienen origen en la Ley Nacional 24.443, promulgada en 1995, por medio de la cual se crea el Fondo de Emergencia Social para el Conurbano de las Ciudades de Rosario y Santa Fe. 
Este Fondo (comúnmente denominado «Fondos del Conurbano») está formado por la afectación de hasta 30 millones de pesos anuales de los recursos que le corresponden a la Provincia de Buenos Aires por la Ley 23.966 del año 1991 (Impuestos/Previsión Social).

La ley 24.443 faculta al Gobierno de la Provincia de Santa Fe como responsable de la administración y disposición del fondo, mediante una Unidad Ejecutora con representación parlamentaria en proporción a las bancas de los distintos partidos. Finalmente, la Unidad Ejecutora Provincial del «Fondo de Emergencia Social» autorizó el financiamiento de $\$ 2.000 .000$ (dos millones de pesos) para ser destinados al PFIC, aprobándose mediante el decreto $\mathrm{N}^{\circ} 1850$ del Gobierno de la Provincia de Santa Fe en el año 2006. Este monto fue destinado a la Secretaría de Estado de Promoción Comunitaria.

f) Modalidad de ejecución

La Secretaría de Estado de Promoción Comunitaria se constituyó en unidad de aplicación del Programa. Esto significa que fue la encargada de la transferencia de los fondos, la aprobación final de los proyectos y del seguimiento y rendición de los mismos. La Unidad Evaluadora de Proyectos Sociales fue la responsable de la selección, el análisis de las solicitudes y la elaboración de los dictámenes o informes sobre el otorgamiento o no del subsidio.

Los proyectos debían presentarse en un formato predeterminado, acompañados de un conjunto de documentación de la institución solicitante, que serían luego enviados a la Unidad Evaluadora, con funcionamiento en la ciudad de Santa Fe. Luego de que la Unidad cumplía su tarea, el expediente comenzaba un circuito en el que pasaba por una serie de instancias técnicas en las cuales se controlaban diferentes aspectos del expediente, tanto relacionados al proyecto como a la situación de las ONGs solicitantes. El circuito fue el siguiente: Registro de ONG, Coordinación Contable, Liquidación de Gastos e Informes, Revisiva, Liquidación de Gastos e Informes, Jurídica, Despacho, Logística, Secretaría Privada y Notificaciones.

Una vez aprobado el expediente por cada una de estas instancias, se firmaba un convenio entre ambas partes -la institución y la Secretaría de Promoción Comunitaria- en el cual se establecían las condiciones que la institución debía cumplir, siendo la más importante la rendición de cuentas dentro de los 60 días de la efectiva percepción de los fondos. Posterior a la firma se efectuaba la liquidación y el pago del subsidio, en forma de depósito en la cuenta bancaria de la institución o la entrega de cheque del Banco de Santa Fe. La ejecución de los proyectos debía comenzar dentro del mes siguiente a la asignación del subsidio.

\section{El punto de partida}

Durante las últimas décadas, nuestro país se vio afectado por la persistencia de un proceso de empobrecimiento que afecta a gran parte de la población, el agravamiento de los problemas sociales, el aumento de la precarización y la exclusión social. La nueva cuestión social aparece profundamente marcada, no sólo por el aumento de la pobreza sino también por la exclusión material, institucional y simbólica de grandes contingentes poblacionales. 
Estos procesos se relacionan con una serie de transformaciones que pueden resumirse en la reforma estructural del Estado: el pasaje del Estado de Bienestar a un Estado mínimo, que se apartó de lo productivo y de la política social universal, con énfasis en la desregulación, privatización y apertura comercial, que llevaron a un cambio abrupto y traumático y a una fuerte redistribución y concentración de poder e ingresos dentro de la sociedad.

Como consecuencia del achicamiento del Estado, asistimos a la emergencia de la sociedad civil como tercer ámbito frente al Estado y el mercado, como portadora de intereses universalizables, expresables en sus organizaciones. El surgimiento y la creciente importancia de las organizaciones de la sociedad civil implica la búsqueda de una recuperación del capital social, entendido como el conjunto de valores compartidos, normas sociales, cultura, tasa de asociacionismo, es decir, la capacidad de construir concertaciones, redes, sinergias, clima de confianza entre los diversos actores sociales y la orientación al trabajo voluntario.

Por otro lado, pasa a ser central el rol de las políticas sociales para atenuar las consecuencias negativas de las políticas de ajuste: empobrecimiento, desempleo, crisis social.

En este contexto surge el PFIC, cuyo objetivo fue «fortalecer las iniciativas comunitarias que tendieran a la Prevención, Promoción y Contención de grupos vulnerables y revalorizar el accionar de las mismas en el ámbito comunitario». Concretamente, el programa propuso «contribuir con las organizaciones, Municipios y Comunas para que las mismas desarrollen acciones comunitarias, promoviendo la participación ciudadana y el desarrollo local».

\section{Aparición en escena}

La ejecución del PFIC en la Ciudad de Rosario comienza a partir de la formación, en el ámbito de la Subsecretaría de Promoción Comunitaria, de un área ad hoc a cargo de un equipo técnico que sería responsable de la implementación del programa en esa localidad ${ }^{2}$. En principio, este equipo estaba conformado por dos Licenciados en Trabajo Social y dos estudiantes avanzadas de Ciencia Política.

Vale aclarar que ya existía dentro del ámbito de la Subsecretaría un área de Proyectos Sociales que, al momento del surgimiento del Programa de Fortalecimiento, se encontraba ejecutando un programa de similares características, el PO.SO.CO. (Políticas Sociales Comunitarias). Las razones esgrimidas por las autoridades acerca de la constitución de un nuevo equipo, cuando ya existía un área encargada de ello, fueron que la competencia territorial de cada programa difería (el Programa de Fortalecimiento en Rosario y el PO.SO.CO. en el conurbano) y que se buscaba una mayor eficiencia en la aplicación. En la práctica no se desarrolló ningún tipo de trabajo en forma articulada entre los miembros de ambas áreas.

Una de las debilidades que se manifestó desde el comienzo de la ejecución del Programa fue la irregularidad con respecto a la situación laboral de los

\footnotetext{
${ }^{2}$ El las otras localidades donde se implementó el PFCI no se conformaron equipos técnicos a cargo del mismo. Los proyectos que de allí surgieran debían ser remitidos directamente a la Unidad Evaluadora que se encontraba en la ciudad de Santa Fe.
} 
miembros del equipo y a la modalidad y plazos de pago por el trabajo, ya que no se generó ningún vínculo legal entre los integrantes del mismo y la Provincia de Santa Fe.

En un principio, el grupo de trabajo actuó «en el aire», sin bases firmes, ya que había un desconocimiento general acerca del Programa por parte de las autoridades. En este sentido, todo el trabajo desarrollado fue un proceso de autoaprendizaje, de «ensayo y error», en el que nunca se recibió capacitación adecuada.

Tampoco se contó con un espacio físico propio ni con los recursos necesarios para llevar a cabo el desenvolvimiento de las tareas. Por lo tanto, nuestra labor se desarrolló en oficinas afectadas a otras dependencias, compartiendo el mobiliario y las herramientas de trabajo, situación que dificultó la tarea.

\section{Inicio del Programa: los primeros pasos}

La difusión del Programa estaba a cargo de otras dependencias dentro de la estructura de Promoción Comunitaria (Gabinete de Asistencia Técnica, Coordinación General, Dirección de Acción Social, Secretaría Privada, Dirección de Tercera Edad). La mayor parte de las instituciones se acercaron a partir de la derivación de estas dependencias; si bien el Programa fue publicado en la página web del gobierno de Santa Fe, no fue difundido masivamente en medios de comunicación.

El equipo tenía como primer objetivo recibir a las instituciones y orientarlas acerca de los lineamientos del Programa y de la documentación solicitada. El principal problema que encontró la puesta en práctica fue la situación de las instituciones en relación a la documentación solicitada, ya que la mayoría de las ONGs interesadas en el subsidio carecían de la mayor parte de la misma. Con el fin de salvar este obstáculo y dinamizar el Programa, comenzó por parte del equipo un trabajo de asesoramiento, que en un principio no estaba contemplado, para gestionar la documentación necesaria. En este marco se incorporó a un abogado al equipo para tornar más efectiva esta labor.

En este sentido, consideramos que antes de poner en ejecución un Programa similar, se hace necesario un trabajo de «asesoramiento técnico» a las ONGs que tenga como objetivo regularizar su situación o bien reconsiderar la documentación a solicitar.

Respecto a la documentación requerida, en la práctica se hizo evidente que fue excesiva y redundante. Esto es confirmado por entrevistas realizadas por el equipo a los representantes de las instituciones encargados de gestionar el subsidio, quienes lo consideraron como uno de los aspectos negativos del Programa y manifestaron que gestionar toda la documentación les resultó «difícil».

Esta percepción se debe al hecho de que las instituciones debieron presentar la misma documentación en varias dependencias de la provincia (registro de ONGs y SIPAF, para obtener o actualizar los respectivos certificados, y para el mismo Programa de Fortalecimiento, que exigía, entre otras, la presentación de estas dos constancias). Esta situación se vería superada si existiera una base de datos de ONGs de la Provincia, que centralizara la información y se actualizara constantemente, siendo accesible para los diferentes organismos provincia- 
les, con el fin de tornar más eficiente la implementación de políticas y programas que tengan como destinatarios a estas instituciones.

Por otra parte, también consideramos innecesaria la solicitud de documentación que ya ha sido aprobada por la Inspección General de Personas Jurídicas, órgano fiscalizador de Asociaciones con Personería Jurídica de la Provincia de Santa Fe, como requisito para otorgar certificado de Personería Jurídica o de Subsistencia de la misma.

El hecho de que la mayor parte de las instituciones carecieran de la documentación, provocó un retraso considerable en el proceso de gestión del subsidio. También tuvo como resultado que una gran cantidad de instituciones que se acercaron con intenciones de gestionarlo vieran finalmente truncas sus intenciones, ya que no contaban con los recursos necesarios (tanto humanos como de tiempo y dinero) para obtener toda la documentación requerida.

Como parte del trabajo que comenzamos a realizar (por iniciativa propia) ante la realidad con que nos encontramos durante la ejecución del Programa, se encuentra la elaboración de los proyectos. Si bien existía un formato predeterminado, muchas instituciones encontraron dificultades a la hora de armarlo, ya que no contaban con los recursos materiales necesarios (una PC) o con un adecuado nivel de preparación para la correcta elaboración del mismo. Con esta afirmación no pretendemos subestimar a las personas que participan en organizaciones comunitarias -al contrario, su saber es muy rico y los miembros del equipo hemos aprendido mucho de ellos- pero una de las falencias fundamentales de este tipo de instituciones es el desconocimiento acerca de la elaboración de proyectos. En este marco, la mayor parte de los proyectos finalmente presentados fueron elaborados por el equipo, a partir de lineamientos proporcionados por las instituciones.

\section{El problema de la centralización administrativa}

La centralización administrativa en la ciudad de Santa Fe también causó importantes demoras. En primer lugar, debido a que los expedientes o la documentación requerida posteriormente a la presentación del proyecto debían ser enviados a la capital provincial, lo que implicó sumar tiempo al proceso total de gestión del mismo. En segundo lugar, a causa de que el equipo a cargo del Programa en Rosario actuó como mero intermediario entre las instituciones y las instancias de evaluación y decisión de Santa Fe, sin capacidad decisoria propia.

Teniendo en cuenta que la comunicación entre las dependencias en ambas ciudades no era personal sino telefónica, y que se realizaba por medio de la Unidad Evaluadora y no directamente con cada instancia, se generaron fallas en la comunicación que dificultaron y entorpecieron el proceso. Por otra parte, la imposibilidad de acceder directamente a los expedientes, ya que se encontraban en la ciudad de Santa Fe, también afectó la gestión. Como ejemplo ilustrativo, se reclamaba documentación que ya había sido presentada en tiempo y forma porque había sido extraviada en la ciudad capital, hecho que se repitió en diversas oportunidades.

A partir de nuestra experiencia, consideramos que urge llevar a cabo la descentralización y la autonomía administrativa de la ciudad de Rosario, con el 
fin de lograr una mayor eficiencia en la puesta en práctica de políticas sociales.

\section{Un largo y sinuoso camino}

En teoría, la función de la Unidad Evaluadora era, como su denominación lo indica, evaluar y aprobar los proyectos y la documentación de las instituciones, detectar y salvar los errores para que el expediente recorriera las sucesivas instancias de control en forma dinámica. No obstante, en la práctica a la mayor parte de los mismos, luego de ser aprobados por esta primera instancia, se le detectaron errores en la documentación presentada (aunque muchas veces estos requerimientos no presentaban bases sólidas), lo que significó un retroceso ya que debían volver a esta primera instancia y, una vez subsanado el error, comenzar nuevamente el circuito. Lógicamente, esta forma de operar extendió el tiempo del proceso.

En muchos casos, debido a esta deficiencia en el sistema de evaluación, nos encontramos con expedientes que, transcurrido un año o más desde su iniciación (tiempo en el que fue controlado y en más de una ocasión por todas las instancias), con sus consiguientes avances y retrocesos, se detectó la falta de algún tipo de documentación.

Por otra parte, una situación que generó demoras en la gestión de los subsidios fue la solicitud de documentación «en forma retroactiva», dentro de la cual encontramos dos modalidades:

1) la solicitud de documentación que, al momento en que las instituciones presentaron el proyecto y fue aprobado no era requerida y que, posteriormente, sí fue incluida y solicitada.

2) documentación que tiene fecha de caducidad y fue presentada en tiempo y forma, pero debido al excesivo tiempo de demora del circuito, dejó de ser válida en el transcurso del mismo, y volvió a ser solicitada.

Esta situación es totalmente cuestionable, ya que las instituciones debieron hacerse responsables por decisiones arbitrarias o por dilación burocrática, y realizar nuevamente la gestión de trámites que implican un gran costo de tiempo y de dinero.

En síntesis, el circuito burocrático que debían realizar los expedientes provocó un retraso en la aprobación de proyectos que atentó contra la efectiva ejecución de los mismos. En este sentido, otro de los aspectos considerados negativos por los representantes institucionales fue la lentitud del proceso desde la presentación del proyecto hasta la entrega del subsidio.

El principal problema en relación a este punto surgió con instituciones que gestionaron proyectos de capacitación y recibieron los fondos en los últimos meses del año. Teniendo en cuenta que durante los meses de enero y febrero se dificulta la ejecución de estos proyectos debido al período vacacional, muchas instituciones no tuvieron otra posibilidad que comenzar las actividades en el mes de marzo, produciéndose un desfasaje entre la fecha de ejecución y el período dentro del cual debían presentar la rendición de cuentas (60 días a partir de la percepción de los fondos), y también incumpliendo una de las reglas del Programa, que establecía que la ejecución debía comenzar dentro del mes posterior a recibido el dinero.

Respecto al largo camino que debieron atravesar los expedientes (no olvi- 
demos que detrás de ellos hay muchas personas involucradas, que depositaron en estos proyectos sus esperanzas: los integrantes de las instituciones, los capacitadores, la población beneficiaria en general), también cabe destacar la rigidez con que fueron observados los reglamentos del Programa por parte de las instancias de control, sobre todo en los últimos meses de gestión del gobierno Justicialista, que en muchas ocasiones atentó contra la eficacia del Programa. Si bien consideramos necesario el cumplimiento de las normas, también creemos que pueden presentarse excepciones ante las cuales es necesario otorgar una mayor flexibilidad, con el objetivo de obtener mejores resultados.

Como ejemplos, dos casos ilustran esta rigidez. Por un lado, uno de los requerimientos era la presentación de títulos de los capacitadores acerca del taller que brindarían. Muchas instituciones presentaron capacitadores que ya brindaban talleres en la misma, en forma sostenida y satisfactoria, y que no contaban con títulos habilitantes ya que el oficio había sido transmitido en el ámbito familiar o algún otro ámbito informal (por ejemplo, capacitadores en talleres de tejido y de música). En estos casos, se presentaban notas de la misma institución o de otras donde el capacitador se había desempeñado, dando cuenta de su idoneidad y capacidad para brindar los talleres, pero no fueron finalmente aprobados por las instancias de control, especialmente por el área Jurídica. A nuestro juicio, en estos casos debía realizarse una excepción, aún más teniendo en cuenta que el Programa apuntó a fortalecer a sectores vulnerables: la mayor parte de las instituciones con fines sociales trabajan con personas que carecen de educación en ámbitos formales y por este motivo se encuentran fuera del mercado de trabajo, encontrando en este tipo de instituciones un espacio donde desarrollar sus oficios, adquiridos en el ámbito familiar o mediante la práctica como un medio de subsistencia.

Otro ejemplo se dio con instituciones muy antiguas, que tras el largo tiempo transcurrido desde su fundación, habían extraviado el acta constitutiva, uno de los documentos requeridos para la presentación de los proyectos. Ante esta situación, el procedimiento a seguir era solicitar una copia de la misma en la Inspección General de Personas Jurídicas, donde era necesario haberla presentado para obtener la personería jurídica. Pero nos encontramos ante el hecho de que en la Inspección tampoco contaban con una copia del documento en cuestión, ya que se había extraviado debido a las reiteradas mudanzas de este organismo u otros motivos accidentales. Tampoco estos casos, a pesar de notas emitidas en las que el organismo manifestaba que no contaba con el documento pero que el mismo había sido presentado en tiempo y forma, es decir que la situación de la institución era regular, fueron contemplados por las instancias de control de Santa Fe, otra vez especialmente por el área de Jurídica. En este sentido, creemos que estas instancias deben atenerse a los dictámenes de la Inspección General, que es el máximo órgano de control de las instituciones con personería jurídica, y no avasallar sus funciones.

Por otra parte, un factor que limitó la eficacia del Programa fue la proximidad del cambio de gobierno ${ }^{3}$. En este marco, la mayor parte de los proyectos presentados con la anterioridad suficiente como para llegar a ser otorgados antes de que se terminara el período de gobierno bajo el cual habían sido gestiona-

${ }^{3}$ Final del mandato del Gobernador Jorge Obeid, el 10/12/07. 
dos, debido a la lentitud de las instancias de control en Santa Fe, no habían recibido el subsidio y continuaban en el circuito al momento en que se realizó el traspaso de gobierno ${ }^{4}$; dado que se desconocía cuál sería el futuro del Programa de Fortalecimiento, esta situación generó incertidumbre y un sentimiento de desilusión en las instituciones afectadas, que luego de mucho tiempo y esfuerzos esperaban recibir el subsidio.

En este sentido, los subsidios no fueron entregados en la cantidad y calidad esperadas. Consideramos que una forma de evitar estas fallas hubiera sido la elaboración de un cronograma de actividades, y su efectivo cumplimiento, estableciendo plazos para la presentación de proyectos, para su aprobación (incluyendo el tiempo máximo que puede permanecer un expediente en cada instancia y en el circuito total), para comenzar la ejecución y el monitoreo de los proyectos. A nuestro juicio, la necesidad de este cronograma era aun más urgente debido a la cercanía del proceso electoral, y el consiguiente cambio de gobierno.

El motivo fundamental que explica la imposibilidad de desarrollar el trabajo de forma previsible y ordenada, organizando un cronograma susceptible de ser cumplido, era la incertidumbre acerca de la duración del circuito que debían realizar los expedientes.

Otra falla del diseño del PFIC fue que no contempló el monitoreo de la ejecución de los proyectos ni una evaluación general del Programa. Si bien se realizaron visitas posteriores a la entrega del subsidio, fueron intermitentes y funcionaron más como forma de mantener el vínculo con las instituciones que como instancia de contralor, ya que no se preveían sanciones ni estaba contemplado un seguimiento periódico y sistemático de la ejecución de los proyectos. Uno de los obstáculos fundamentales encontrados para realizar visitas en forma periódica fue la falta de medios para la movilidad del equipo.

Este punto es de fundamental importancia, ya que permite verificar si las instituciones cumplieron con lo establecido en el convenio, si el proyecto se fue desarrollando de forma correcta y si los fondos recibidos fueron utilizados para los fines solicitados, es decir, si los recursos del programa se utilizaron de manera adecuada.

En cuanto a la evaluación, nunca estuvo contemplada en el diseño. La misma surgió como una iniciativa del propio equipo de la ciudad de Rosario, mientras que en el resto de las localidades en las cuales se implementó el Programa no se efectuó evaluación alguna por parte del organismo ejecutor ${ }^{5}$.

Otro aspecto problemático fue la modalidad de rendición, en particular para las instituciones que desarrollaron proyectos de capacitación, cuya duración era de seis meses. La rendición debía presentarse dentro de los 60 días a partir de la percepción de los fondos y por la totalidad del monto recibido. En el caso de pago a capacitadores, debían presentarse recibos o facturas de los mismos. Para cumplir con esta exigencia se presentaban dos opciones: abonar a los capacitadores los seis meses juntos al inicio de la ejecución del proyecto, quedando la institución en una situación de riesgo ya que existía la

${ }^{4}$ Fueron presentados, en la ciudad de Rosario, un total de 47 (cuarenta y siete) proyectos, de los cuales 26 (veintiséis) recibieron efectivamente el subsidio y los 31 (treinta y uno) restantes aun continuaban en el circuito.

${ }^{5}$ Por este motivo no se puede establecer una comparación con el impacto que tuvo el PFIC en el resto de las localidades. 
posibilidad de que los capacitadores, una vez recibido el dinero, no cumplieran con su trabajo; o la situación contraria, es decir, que los talleristas emitieran recibos o facturas por el monto total a cobrar en los seis meses, sin haberlo percibido, quedando de esta forma expuestos a que el pago no se hiciera efectivo.

Esta situación se vio agravada debido a la ausencia de monitoreo y de aplicación de efectivas sanciones a instituciones o personas que incumplieran lo acordado -si bien el incumplimiento de la rendición de cuentas quedaría alcanzado por la ley de contabilidad de la provincia, hemos comprobado que ante retrasos o incumplimientos no se han hecho efectivas las sanciones previstas. De esta forma, a fin de cumplir con el tiempo establecido para presentar la rendición, las instituciones y los capacitadores debieron acordar, jugando un rol fundamental la confianza mutua.

Una modalidad más adecuada de rendición para los proyectos de capacitación sería mensualmente, para evitar este tipo de situaciones que generan incertidumbre, o bien la emisión de cheques de pago diferido a fecha.

Algunas instituciones, que recibieron el subsidio en los últimos meses del año 2007, y debido a la ya mencionada dificultad para ejecutar proyectos de capacitación en período vacacional, debieron solicitar una prórroga para presentar la rendición, dado que aquellos comenzarían a desarrollarse a partir de marzo de 2008.

\section{¿Se logró el fortalecimiento institucional?}

Los Programas de Fortalecimiento deberían servir para la construcción de capacidades ciudadanas y para ampliar el capital social de los sectores excluidos. Fortalecer el tejido local significa fortalecer y cualificar los espacios e instancias de participación local, como escenarios de construcción del desarrollo social local e impulsar iniciativas cogestionadas con la comunidad en esa materia.

Los objetivos del Programa apuntaron a aumentar la capacidad de las organizaciones comunitarias en cuanto a su carácter de mediadoras y nexo natural entre el Estado y la sociedad, y a proveer las herramientas necesarias que les posibilitara la implementación de iniciativas que promuevan la participación comunitaria y el desarrollo local, revalorizando su accionar en el ámbito comunitario.

En un nivel más concreto, si el objetivo del Programa era «fortalecer»las instituciones, significa que sus efectos positivos deberían prolongarse más allá de la finalización de la ejecución. ¿Fue cumplido este objetivo? La respuesta a esta pregunta debe tener en cuenta las diferencias entre los proyectos presentados. En el caso de construcciones o adquisición de equipamiento, la respuesta es afirmativa, ya que son mejoras duraderas en el tiempo, que fortalecen a las instituciones en el sentido de que permiten aumentar la cantidad y calidad de sus actividades. En estos casos ocurre un fortalecimiento que podríamos denominar «material». En el caso de proyectos de capacitación, el fortalecimiento es cuestionable, ya que estos talleres sólo duraron seis meses. En este marco se plantean muchos interrogantes: ¿se fortaleció a la institución o a los capacitados?, ¿las instituciones pueden seguir brindando estos talleres 
por su propia cuenta, sin solicitar subsidios?, ¿es posible que este Programa se extienda?, ¿cómo sostener en el mediano y largo plazo los procesos participativos?

Muchas iniciativas de promoción de la participación social se abandonan por diferentes motivos, pero principalmente por el cese de la voluntad y el compromiso político del Estado para continuarlas. Los cambios de funcionarios, de gobierno, los problemas presupuestarios y otros motivos de similar naturaleza han truncado experiencias valiosas, que luego de promover fuertes procesos de participación social, no han sabido o no han podido generar procesos graduales de reducción de aportes y estrategias de fortalecimiento de capacidades locales para el sostén financiero y social de los procesos iniciados.

La única manera de que dichos procesos sean verdaderamente sostenidos en el tiempo, es logrando autonomía de los espacios de participación de las frecuentes e impredecibles fluctuaciones político/institucionales. Esta autonomía podrá lograrse a condición de que se refuerce la institucionalización de tales espacios y se traspase el poder de conducción (gestión) de los mismos desde el Estado hacia la sociedad civil local.

Pero para lograr esta meta es necesario un previo proceso de acompañamiento por parte de los organismos estatales, que sea capaz de superar la participación «ficticia», generada como requisito para acceder a algunos fondos, y que no resulta sustentable en el tiempo y acaba cuando los fondos se terminan. Si los procesos de construcción colectiva participativa no son verdaderamente genuinos, propios, autoconvocados y autogestionados, los participantes dejan paulatinamente de pertenecer a ellos, se desaniman y los abandonan.

\section{Lo que no fue}

Creemos que el Programa de Fortalecimiento, más allá de las limitaciones propias de un programa social focalizado, tuvo potencialidades que no fueron desplegadas, debido a la falta de recursos y a la escasa voluntad política.

A medida que se desarrolló nuestro trabajo, nos fuimos planteando como objetivo posibilitar la comunicación y la interacción entre las instituciones con las que trabajamos, es decir, facilitar su trabajo en red. Consideramos que esta forma de trabajo, tan en boga en los últimos tiempos, es la más adecuada para lograr un verdadero fortalecimiento de las instituciones. Una red es el resultado de la conjunción de esfuerzos, en donde se establecen relaciones de cooperación horizontal; es una forma de organización que permite el intercambio y la producción conjunta de conocimientos y su actividad y existencia depende de la iniciativa de cada una de sus partes o nódulos y de la instancia central de coordinación; se caracteriza por su flexibilidad y la capacidad de adaptarse a situaciones cambiantes del contexto.

Las redes proporcionan un mecanismo participativo en el que las organizaciones tienen la oportunidad de compartir experiencias, metodologías, conocimientos, etc., para con ello fortalecerse y en conjunto incrementar el impacto de sus actividades respectivas; en este sentido nuestro deseo era propiciar los mecanismos y el ambiente adecuado para crearlas.

La escasez tanto de recursos materiales como de tiempo necesario para 
realizar este trabajo, así como la falta de apoyo de parte de las autoridades, impidieron el logro de este objetivo. El verdadero y real fortalecimiento institucional se hubiera producido con la creación de esta red.

\section{Bibliografía}

BRIONES, G. Evaluación de Programa Sociales. Editorial Trillas, México, 1991.

GARCÍA DELGADO, D. Organizaciones de la sociedad civil y política social. El problema de la articulación. Primer Congreso Nacional de Políticas Sociales, Universidad Nacional de Quilmes, mayo de 2002. http://ps.unq.edu.ar/

MOKATE, K. Convirtiendo al «monstruo» en aliado: la evaluación como herramienta de la gerencia social. Instituto Interamericano para el Desarrollo Social -INDES- Abril 2000.

WILDE, J.; SOCKEI, S. Manual de Evaluación. Fondo Editorial de la Universidad de Albuquerque, 1995.

INTERNET

www.santafe.gov.ar 